\title{
FINITE GROUPS OF DIFFEOMORPHISMS ARE TOPOLOGICALLY DETERMINED BY A VECTOR FIELD
}

\author{
F.J. TURIEL AND A. VIRUEL
}

\begin{abstract}
In a previous work it is shown that every finite group $G$ of diffeomorphisms of a connected smooth manifold $M$ of dimension $\geq 2$ equals, up to quotient by the flow, the centralizer of the group of smooth automorphisms of a $G$-invariant complete vector field $X$ (shortly $X$ describes $G$ ). Here the foregoing result is extended to show that every finite group of diffeomorphisms of $M$ is described, within the group of all homeomorphisms of $M$, by a vector field.
\end{abstract}

As a consequence, it is proved that a finite group of homeomorphisms of a compact connected topological 4-manifold, whose action is free, is described by a continuous flow.

\section{INTRODUCTION}

The study of the automorphism group, or centralizer, of a complete vector field $X$ of class $C^{r}, r \geq 1$, or more precisely that of its quotient by the flow of $X$, is a classical question with a great amount of interesting results. Often these quotient groups are trivial or almost trivial if a reasonable hypothesis of transversality is imposed.

Therefore it is natural to consider the inverse point of view (the inverse Galois problem): given a group of diffeomorphisms $G$ do construct a complete vector field $X$ whose automorphism group, up to quotient by the flow, equals $G$ (shortly one will say that $X$ determines or describes $G$ ). Notice that this last problem can be addressed in topological manifolds and homeomorphisms by replacing the vector field by a continuous flow.

In [8] it is shown that every finite group of diffeomorphisms of a smooth connected manifold is determined, within the group of all diffeomorphisms, by a vector field. Here the foregoing result is extended to show that every finite group of diffeomorphisms can be described, within the group of all homeomorphisms, by a vector field.

Theorem 1.1. Let $M$ be a connected $C^{\infty}$ manifold of dimension $m \geq 2$, and $G$ be a finite subgroup of diffeomorphisms of $M$. Then there exists $X$, a complete $G$-invariant vector field 
on $M$, such that the map

$$
\begin{aligned}
G \times \mathbb{R} & \rightarrow \operatorname{Aut}_{0}(X) \\
(g, t) & \mapsto g \circ \Phi_{t}
\end{aligned}
$$

is a group isomorphism, where $\Phi$ and $\operatorname{Aut}_{0}(X)$ denote the flow and the group of continuous automorphisms of $X$ respectively.

An immediate consequence of Theorem 1.1 is that any smoothable finite group of homeomorphisms of a connected topological manifold is determined by a continuous flow.

Corollary 1.2. Let $G$ be a smoothable finite group of homeomorphisms of a connected topological manifold $E$ of dimension $\geq 2$. Then there exists a $G$-invariant continuous flow $\psi: \mathbb{R} \times E \rightarrow E$ such that the map

$$
\begin{aligned}
G \times \mathbb{R} & \rightarrow \operatorname{Aut}_{0}(\psi) \\
(g, t) & \mapsto g \circ \psi_{t}
\end{aligned}
$$

is a group isomorphism, where $\operatorname{Aut}_{0}(\psi)$ denotes the group of continuous automorphisms of $\psi$.

Remark 1.3. While it is a classical result that any finite group of homeomorphisms of a compact surface is smoothable, the situation in dimension three is not straightforward: not every finite group $G$ of homeomorphisms of a 3-dimensional topological compact manifold is smoothable [2]. Indeed $G$ is smoothable if and only if it is locally linear [6, Theorem 2.1 and Remark 2.4].

Therefore the corollary above applies to connected compact surfaces and, in the case of topological connected compact 3-manifolds, if $G$ is locally linear.

For a generalization of Corollary 1.2 to some cases of non-smoothable actions see Example 6.4 and Theorem 6.5. In this last one we show that a finite group of homeomorphisms of a compact connected topological 4-manifold, whose action is free, is described by a continuous flow.

Terminology: We assume the reader is familiarized with our previous paper [8]. All structures and objects considered in this work are smooth, i.e. real $C^{\infty}$, and manifolds are without boundary, unless another thing is stated. Whenever we say a set is countable we mean the set is either a finite set or a countably infinite set. For the general questions on Differential 
Geometry the reader is referred to [5] while we refer to [3] for basic facts on Differential Topology.

\section{Some PRELIMINARY NOTIONS}

Given a vector field $Z$ on an $m$-manifold $M$, a continuous automorphism of $Z$ is a homeomorphism $f: M \rightarrow M$ which maps integral curves of $Z$ into integral curves of $Z$ (i.e. if $\gamma(t)$ is an integral curve of $Z$ then $f(\gamma(t))$ is so.) The set $\operatorname{Aut}_{0}(Z)$ of all continuous automorphisms of $Z$ is a subgroup of the group of homeomorphisms of $M$. If $Z$ is complete and $\Phi_{t}$ denotes its flow, then $f \in \operatorname{Aut}_{0}(Z)$ if and only if $f \circ \Phi_{t}=\Phi_{t} \circ f$ for any $t \in \mathbb{R}$.

In a more general setting, given a topological space $E$ a continuous flow is a continuous $\operatorname{map} \psi: \mathbb{R} \times E \rightarrow E$ such that $\psi_{0}=I d$ and $\psi_{t+s}=\psi_{t} \circ \psi_{s}$ for each $t, s \in \mathbb{R}$. As before, $\operatorname{Aut}_{0}(\psi)$ is the group of all homeomorphisms $f: E \rightarrow E$ such that $f \circ \psi_{t}=\psi_{t} \circ f, t \in \mathbb{R}$. We say that a subset $S$ of $\operatorname{Homeo}(E)$ is smoothable if there exists a structure of smooth manifold on $E$ which is compatible with the preexisting topology and makes every element of $S$ a diffeomorphism.

Returning to the smooth framework again, given a vector field $Z$ on an $m$-manifold $M$, a pseudo-circle of $Z$ is a subspace of $M$ which is homeomorphic to $S^{1}$ and consists of a regular trajectory of $Z$ and an isolated singularity. In this case the $\alpha$-limit and the $\omega$-limit of the regular trajectory is the singular point.

Let $B(r)$ be the open ball in $\mathbb{R}^{m}$ centered at the origin and radius $r>0$. For the purpose of this work, we will say that $p \in M$ is a source of $Z$ if there exists an open neighborhood of this point which is diffeomorphic to an open ball $B(r)$, with $p \equiv 0$, such that in the coordinates given by the diffeomorphism

$$
Z=\varphi \cdot\left(\sum_{j=1}^{m} x_{j} \frac{\partial}{\partial x_{j}}\right)
$$

where

(1) $\varphi$ is a non-negative function and $\varphi^{-1}(0)$ is countable, and

(2) on each ray issuing from the origin there are at most a finite number of zeros of $\varphi$.

By condition (2), for every ray issuing from the origin there exists just one regular trajectory whose $\alpha$-limit is $p \equiv 0$ and that near the origin lies along this ray.

A point $q \in M$ is called a rivet if the following hold:

(a) $q$ is an isolated singularity of $Z$, 
(b) around $q$ one has $Z=\psi \tilde{Z}$ where $\psi$ is a function and $\tilde{Z}$ a vector field with $\tilde{Z}(q) \neq 0$, and

(c) no trajectory has $q$ as $\alpha$-limit and $\omega$-limit at the same time.

Note that by (b) and (c), any rivet is the $\omega$-limit of exactly one regular trajectory, the $\alpha$-limit of another different one and moreover, it is an isolated singularity of index zero.

A topological rivet means an isolated singularity of $Z$ that is the $\alpha$-limit of a single regular trajectory, the $\omega$-limit of another single regular trajectory and both trajectories are different. As one would expect, any rivet is a topological rivet.

By definition, a chain of $Z$ is a finite and ordered sequence of three or more different regular trajectories, each of them called a link, such that:

(a) The $\alpha$-limit of the first link is a source or empty.

(b) The $\omega$-limit of the last link is a pseudo-circle.

(c) Between two consecutive links the $\omega$-limit of the first one equals the $\alpha$-limit of the second one. Moreover this set consists in a rivet.

The number of links defining a chain is called the order of the chain. The $\omega$-limit of a chain is that of its last link.

Given a subset $Q \subset M$, we say that the dimension of $Q$ does not exceed $\ell$, or $Q$ can be enclosed in dimension $\ell$, if there exists a countable collection $\left\{N_{\lambda}\right\}_{\lambda \in L}$ of submanifolds of $M$, all of them of dimension $\leq \ell$, such that $Q \subset \bigcup_{\lambda \in L} N_{\lambda}$. Note that the countable union of sets whose dimension does not exceed $\ell$, does not exceed dimension $\ell$ too. On the other hand, if the dimension of $Q$ does not exceed $\ell<m$ then $Q$ has measure zero and therefore $Q$ has empty interior.

Let us give the last definition of this section. A vector field $Z$ on $M$ is called limit (abbreviation of "with an almost controlled $\omega$-limit") if the following conditions hold:

(i) The set of zeros of $Z$ is discrete (that is with no accumulation point).

(ii) $Z$ has exactly one pseudo-circle.

(iii) There exists a set $Q \subset M$ whose dimension does not exceed $m-1$ such that the trajectory of any point of $M-Q$ has the pseudo-circle as $\omega$-limit.

(iv) $Z$ has no chain and no periodic regular trajectory.

By (iii) the union of the trajectory of any point of $M-Q$ and the pseudo-circle is a connected set, hence the $Z$-saturation of $M-Q$ together with the pseudo-circle is a connected 
set too. Therefore $M=\overline{M-Q}$ is connected. Moreover $\operatorname{dim} M \geq 2$, otherwise $M$ equals the pseudo-circle and (iii) cannot hold.

Proposition 2.1. Each sphere $S^{k}, k \geq 2$, supports a limit vector field.

Proof. On $S^{k} \subset \mathbb{R}^{k+1}$ consider the vector field

$$
\xi=-\sum_{j=1}^{k} x_{j} x_{k+1}\left(\partial / \partial x_{j}\right)+\left(1-x_{k+1}^{2}\right)\left(\partial / \partial x_{k+1}\right),
$$

orthogonal projection of the vector field $\partial / \partial x_{k+1}$ onto the sphere, whose trajectories go from the south pole to the north one. Since $\xi$ is transverse to the equator $E=\left\{x \in S^{k} \mid x_{k+1}=0\right\}$, one may identify an open neighborhood $A$ of $E$ to $(-\varepsilon, \varepsilon) \times S^{k-1}$, endowed with coordinates $(t, y)=\left(t, y_{1}, \ldots, y_{k}\right)$ where $S^{k-1} \subset \mathbb{R}^{k}$, in such a way that $E$ corresponds to $\{0\} \times S^{k-1}$ and $\xi=\partial / \partial t$. Thus the north band is given by $t>0$ and the south one by $t<0$.

Let $\varphi: \mathbb{R} \rightarrow \mathbb{R}$ be a function such that $\varphi((-\infty,-\varepsilon / 2])=1, \varphi([\varepsilon / 2, \infty))=-1$, and $\varphi(t)=0$ if and only if $t=0$.

First assume $k=2$. On $A$ consider the vector field $Z^{\prime}=\varphi(t) \partial / \partial t+\left(1-\varphi^{2}(t)\right)\left(-y_{2} \partial / \partial y_{1}+\right.$ $\left.y_{1} \partial / \partial y_{2}\right)$ and extend it outside $A$ by $-\xi$ on the north part and by $\xi$ on the south one. Fixed a point $p_{0} \in E$, consider a function $\psi: S^{2} \rightarrow \mathbb{R}$ vanishing at $p_{0}$ and positive on $S^{2}-\left\{p_{0}\right\}$. It is easily checked that $Z=\psi Z^{\prime}$ is a limit vector field (here $Q$ is the equator plus both poles, thus the dimension of $Q$ does not exceed 1, and observe that the only sources are the poles).

Now assume $k \geq 3$; let $\widetilde{Z}$ be a limit vector field on $S^{k-1}$ constructed by induction. On $A$ consider the vector field $Z=\varphi(t) \partial / \partial t+\left(1-\varphi^{2}(t)\right) \widetilde{Z}$, where $\widetilde{Z}$ is regarded as a vector field on $A$ in the obvious way, that is tangent to the second factor, and extend it outside of $A$ by $-\xi$ on the north part and by $\xi$ on the south one. We now prove that $Z$ is a limit vector field on $S^{k}$.

First note that the only sources of $Z$ are the poles. Moreover, $Z$ does not have any rivet, which implies that $Z$ has no chain. Indeed clearly no point of $S^{k}-E$ is a rivet; on the other hand if $(0, q)$ is a rivet, as $Z$ is tangent to $\{0\} \times S^{k-1}$ the only trajectory whose $\omega$-limit is this point is included in $\{0\} \times S^{k-1}$. But clearly $(-\delta, 0) \times\{q\}$ for a $\delta>0$ sufficiently small is included in a trajectory with $\omega$-limit $(0, q)$ what leads to contradiction.

By construction, no trajectory in $S^{k}-E$ is regular and periodic, so $Z$ does not possess any periodic regular trajectory. On the other hand, if $\widetilde{Z}$ is regarded as a vector field on $E$ and $\widetilde{Q} \subset E$ satisfies condition (iii) for $\widetilde{Z}$, it suffices to take as $Q$ the union of all trajectories 
of $\xi$ passing through $\widetilde{Q}$ plus both poles. For if $p \in\left(S^{n}-E\right)$ and $q \in E$ belong to the same $\xi$-trajectory, then the $\omega$-limit of the $Z$-trajectory of $p$ equals the $\omega$-limit of the $\widetilde{Z}$-trajectory of $q$.

\section{The Almost Free CASE}

Let $M$ be a connected manifold of dimension $m$. Given a diffeomorphism $\varphi: M \rightarrow M$, the isotropy of $\varphi$ is the set $I_{\varphi}:=\{p \in M: \varphi(p)=p\}$. A point $p \in I_{\varphi}$ is said to be positive or negative according to the sign of the determinant of $\varphi_{*}(p): T_{p} M \rightarrow T_{p} M$. Obviously $I_{\varphi}=I_{\varphi}^{+} \cup I_{\varphi}^{-}$where the positive isotropy $I_{\varphi}^{+}$is the set of positive points and the negative isotropy $I_{\varphi}^{-}$that of negative ones.

If $\varphi \neq I d$ has finite order, that is to say $\varphi$ spans a finite subgroup of diffeomorphisms, then $\varphi$ is an isometry for some Riemannian metric. Thus if $p \in I_{\varphi}$, the use of normal coordinates with origin $p$ allows us to identify the diffeomorphism $\varphi$ with an element of $O(m)$ different from the identity. Therefore locally $I_{\varphi}^{-}$is a regular submanifold of codimension $\geq 1$ and $I_{\varphi}^{+}$ a regular submanifold of codimension $\geq 2$.

By definition the maximal isotropy $I_{\varphi}^{\max }$ is the set of those points $p \in I_{\varphi}^{-}$such that the codimension of $I_{\varphi}^{-}$at $p$ equals 1 . It is easily seen that $I_{\varphi}^{\max }$ is either empty or a closed regular submanifold of codimension 1 . Notice that if $p \in I_{\varphi}^{\max }$ then in normal coordinates with origin $p$ the diffeomorphism $\varphi$ is a symmetry with respect to a hyperplane (the trace of $I_{\varphi}^{\max }$ ).

Let $G$ be a finite group of diffeomorphisms of $M$. Let $e \in G$ be the identity element and $\ell$ be the order of $G$. By the isotropy, the positive isotropy, the negative isotropy and the maximal isotropy of $G$ we mean

$$
I_{G}^{*}=\bigcup_{g \in G-\{e\}} I_{\varphi}^{*}
$$

where $*$ equals nothing,,+- or max respectively.

For the purpose of this work one will say that the action of $G$ is almost free if $I_{G}=I_{G}^{\max }$.

Lemma 3.1. Assume $I_{G}^{+}=\emptyset$. Then the following hold:

(a) If $g$ and $h$ are two different elements of $G-\{e\}$ then $I_{g}^{-} \cap I_{h}^{-}=\emptyset$.

(b) If $I_{g}^{-} \neq \emptyset$ then $g^{2}=e$.

Proof. (a) If $p \in I_{g}^{-} \cap I_{h}^{-}$then $p$ belongs to the positive isotropy of $g h^{-1}$, so $g h^{-1}=e$. (b) If $p \in I_{g}^{-}$then $p$ belongs to $I_{g^{2}}^{+}$, hence $g^{2}=e$. 
In the remainder of this section the action of $G$ is assumed to be almost free. Our goal will be to prove the main theorem under this supplementary hypothesis.

The proof consists of four steps. In the first one, we construct a vector field $Z$ as the gradient of a suitable $G$-invariant Morse function $\mu$. In a second step, one modifies $Z$ for obtaining a new $G$-invariant vector field $Y$ with as many pseudo-circle as (local) minima of $\mu$.

The third part is the construction from $Y$ of a $G$-invariant vector field $X$ that possesses a countable family of chains. These chains are topological invariant of $X$ and allow us to control its continuous automorphisms. Finally, the fourth step is devoted to determine these automorphisms.

3.1. The gradient vector field. Let $\mu: M \rightarrow \mathbb{R}$ be a Morse function that is $G$-invariant, proper and non-negative, whose existence is assured by a result of Wasserman [9]. Let $C$ denote the set of critical points of $\mu$, which is closed, discrete and countable. As $M$ is paracompact, there exists a locally finite family of disjoint open sets $\left\{A_{p}: p \in A_{p}\right\}_{p \in C}$ which is $G$-invariant, i.e. $A_{g \cdot p}=g \cdot A_{p}$ for any $p \in C$ and any $g \in G$. By shrinking each $A_{p}$ if necessary, one constructs a collection of charts $\left\{\left(A_{p}, \rho_{p}\right)\right\}_{p \in C}$ such that:

(C.1) $\rho_{p}\left(A_{p}\right)=B\left(2 r_{p}\right)$ for some $r_{p}>0$ and $\rho_{p}(p)=0$.

(C.2) $\mu=\sum_{j=1}^{k} x_{j}^{2}-\sum_{j=k+1}^{m-1} x_{j}^{2}+\varepsilon x_{m}^{2}+\mu(p)$ on $A_{p}$ where $\varepsilon= \pm 1$. (Of course $k$ and $\varepsilon$ depend on $p$, and $x=\left(x_{1}, \ldots, x_{m}\right)$ are the coordinates associated to the chart $\left(A_{p}, \rho_{p}\right)$. Nevertheless, in order to avoid an over-elaborated notation, these facts are not indicated unless it is completely necessary.)

(C.3) $\rho_{g \cdot p} \circ g \circ \rho_{p}^{-1}$ equals

- the identity map, if the $G$-orbit of $p$ has exactly $\ell$ elements, or

- the identity or the symmetry $\Gamma(x)=\left(x_{1}, \ldots, x_{m-1},-x_{m}\right)$, if the $G$-orbit of $p$ has less than $\ell$ elements.

(C.4) The function $\mu$ can be chosen in such a way that the $G$-orbit of every (local) minimum has $\ell$ elements.

Indeed, let $\mathcal{O} \subset C$ be a $G$-orbit and fix a point $p \in \mathcal{O}$. If $|\mathcal{O}|=\ell$ one constructs such a chart around $p$ and then use the $G$-action to get a chart around any point of $\mathcal{O}$. 
If $|\mathcal{O}|<\ell$ then $p \in I_{G}$ and, by Lemma $3.1,|\mathcal{O}|=\ell / 2$ and there exists just one element $h \in G-\{e\}$ such that $h \cdot p=p$. Moreover $h^{2}=e$. As $I_{h}=I_{h}^{\max }$ there are coordinates $\left(y_{1}, \ldots, y_{m}\right)$ around $p \equiv 0$ such that $h$ is given by the symmetry $\Gamma(y)=\left(y_{1}, \ldots, y_{m-1},-y_{m}\right)$.

By Lemma 7.1 applied to coordinates $\left(y_{1}, \ldots, y_{m}\right)$ (observe that now $x$ and $y$ have exchanged their roles) there exist coordinates $\left(x_{1}, \ldots, x_{m}\right)$ around $p \equiv 0$ in which $h$ is still given by the symmetry $\Gamma(x)=\left(x_{1}, \ldots, x_{m-1},-x_{m}\right)$ and $\mu=\sum_{j=1}^{k} x_{j}^{2}-\sum_{j=k+1}^{m-1} x_{j}^{2}+\varepsilon x_{m}^{2}+\mu(p)$, $\varepsilon= \pm 1$. Now for another point $q \in \mathcal{O}$ choose a $g \in G-\{e\}$ such that $q=g \cdot p$ and set $\rho_{q}=\rho_{p} \circ g^{-1}$.

Finally if our $p$ is a minimum of $\mu$, always with a $G$-orbit of $\ell / 2$ elements, by applying Proposition 7.2 to a $0<r_{p}^{\prime}<\min \left\{1, r_{p}\right\}$ we may modify $\mu$ inside $\rho^{-1}\left(B\left(r_{p}\right)\right)$ to construct a new $h$-invariant Morse function, still called $\mu$, such that each one of its minima in $A_{p}$ is not $h$-invariant and, as before, transfer this modification to every $A_{q}, q \in \mathcal{O}-\{p\}$, by means of a $g \in G-\{e\}$ such that $g \cdot p=q$.

As every minimum in $A_{p}$ of the new $\mu$ is not $h$-invariant, then the $G$-orbit of any minimum in $\bigcup_{q \in \mathcal{O}} A_{q}$ of the new $\mu$ has $\ell$ elements. Obviously the same thing can be done with any other $G$-orbit with $\ell / 2$ elements consisting of minima.

On the other hand since $|\tau(x)| \leq\|x\|^{2}$ in Proposition [7.2, the new function $\mu$ is proper and low bounded by -1 (more exactly the difference between the new function $\mu$ and the old one takes its values in $[-2,0])$. Therefore replacing $\mu$ by $\mu+1$ shows (C.4).

On $M$ there always exists a Riemannian metric $g^{\prime}$ that on each $\rho_{p}^{-1}\left(B\left(r_{p}\right)\right)$ is written as $2 \sum_{j=1}^{m} d x_{j} \otimes d x_{j}$. Therefore shrinking every $A_{p}$ allows to assume $g^{\prime}=2 \sum_{j=1}^{m} d x_{j} \otimes d x_{j}$ on the whole $A_{p}$. Moreover taking into account Property (C.3) of the collection $\left\{\left(A_{p}, \rho_{p}\right)\right\}_{p \in C}$ we may assume, without losing the property above, that $g^{\prime}$ is $G$-invariant by considering $(1 / \ell) \sum_{h \in G} h^{*}\left(g^{\prime}\right)$ instead of $g^{\prime}$ if necessary.

Let $Z^{\prime}$ be the gradient vector field of $\mu$ with respect to $g^{\prime}$ and $\varphi: M \rightarrow \mathbb{R}$ be a $G$-invariant proper function that is constant around every $p \in C$. As before, $\varphi$ can be supposed constant on each $A_{p}$ by shrinking these open sets if necessary. It is well known that the vector field $Z=e^{-\left(Z^{\prime} \varphi\right)^{2}} Z^{\prime}$ is complete. Moreover $Z$ is the gradient of $\mu$ with respect to the $G$-invariant Riemannian metric $\tilde{g}=e^{\left(Z^{\prime} \varphi\right)^{2}} g^{\prime}$. 
On the other hand $\tilde{g}=g^{\prime}$ on every $A_{p}, p \in C$, since $\varphi$ is constant on these sets. Hence

$$
Z=\sum_{j=1}^{k} x_{j} \frac{\partial}{\partial x_{j}}-\sum_{j=k+1}^{m-1} x_{j} \frac{\partial}{\partial x_{j}}+\varepsilon x_{m} \frac{\partial}{\partial x_{m}}
$$

$\varepsilon= \pm 1$ on each $A_{p}$

3.2. Construction of pseudo-circles. Since $\mu$ is non-negative and proper, the $\alpha$-limit of any regular trajectory of $Z$ is a (local) minimum or a saddle of $\mu$, whereas its $\omega$-limit is empty, a (local) maximum or a saddle of $\mu$. Moreover $Z$ does not possesses any pseudo-circle because no trajectory of a gradient vector field has its $\alpha$-limit equal to its $\omega$-limit. Clearly $Z$ does not have rivets nor topological rivets.

Now by modifying $Z$ we will construct a new vector field with as many pseudo-circle as minima of $\mu$.

Let $I$ be the set of minima of $\mu$ and $\tilde{I}$ be that of maxima. For sake of simplicity let us identify $A_{i}$ with $B\left(2 r_{i}\right)$. Denote by $E_{i}, i \in I$, the sphere in $A_{i}$ of radius $r_{i}$ and center the origin. For each $i \in I$ there exist $\varepsilon_{i}>0,0<r_{i}^{\prime \prime}<r_{i}<r_{i}^{\prime}<2 r_{i}$ and a diffeomorphism identifying $\widetilde{A}_{i}=B\left(r_{i}^{\prime}\right)-\bar{B}\left(r_{i}^{\prime \prime}\right)$ with $\left(-\varepsilon_{i}, \varepsilon_{i}\right) \times S^{m-1}$, endowed with coordinates $(t, y)$, in such a way that $E_{i}$ corresponds to $\{0\} \times S^{m-1}$ and $Z$ to $\partial / \partial t$.

Let $\varphi_{i}: \mathbb{R} \rightarrow \mathbb{R}$ be a function such that $\varphi_{i}\left(\left(-\infty,-\varepsilon_{i} / 2\right]\right)=1, \varphi_{i}\left(\left[\varepsilon_{i} / 2, \infty\right)\right)=-1$ and $\varphi_{i}(t)=0$ if and only if $t=0$. On every $\widetilde{A}_{i} \equiv\left(-\varepsilon_{i}, \varepsilon_{i}\right) \times S^{m-1}$ define

$$
Y=\varphi_{i}(t) \partial / \partial t+\left(1-\varphi_{i}^{2}(t)\right) Z_{i}^{\prime}
$$

where:

(1) If $m \geq 3$ then $Z_{i}^{\prime}$ is a limit vector field on $S^{m-1}$ regarded on $\left(-\varepsilon_{i}, \varepsilon_{i}\right) \times S^{m-1}$ in the obvious way.

(2) If $m=2$ then $Z_{i}^{\prime}=\lambda_{i}(t, y)\left(-y_{2} \partial / \partial y_{1}+y_{1} \partial / \partial y_{2}\right)$ where the function $\lambda_{i}:\left(-\varepsilon_{i}, \varepsilon_{i}\right) \times$ $S^{1} \rightarrow \mathbb{R}$ vanishes at some point $\left(0, q_{i}\right)$ of $\{0\} \times S^{1}$ and is positive elsewhere.

Now prolong $Y$ to each $A_{i}$ by $Z$ inside of $A_{i}-\widetilde{A}_{i}$, that is on $\bar{B}\left(r_{i}^{\prime \prime}\right)$, and by $-Z$ outside of $A_{i}-\widetilde{A}_{i}$, that is on $A_{i}-B\left(r_{i}^{\prime}\right)$. In turn, extend $Y$ already defined on $\bigcup_{i \in I} A_{i}$ to the whole manifold $M$ by $-Z$ on $M-\bigcup_{i \in I} A_{i}$

To be sure that $Y$ is $G$-invariant, in each orbit $\mathcal{O}$ included in $I$ choose a point $i$ and construct $Y$ on $A_{i}$ as before. Then by means of the $G$-action construct $Y$ on every $A_{j}, j \in \mathcal{O}$. 
As the action of $G$ on $\bigcup_{j \in \mathcal{O}} A_{j}$ is free by property (C.4) of the family $\left\{\left(A_{p}, \rho_{p}\right)\right\}_{p \in C}$, this construction is coherent. Therefore from now on $Y$ will be assumed $G$-invariant.

Notice that the singularities of $Y$ in $M-\bigcup_{i \in I} E_{i}$ are saddles or sources. The singularities of $Y$ in $\bigcup_{i \in I} E_{i}$ are never sources nor rivets since each of them is the $\omega$-limit of two or more regular trajectories traced in $M-\bigcup_{i \in I} E_{i}$. Thus $Y$ has no topological rivet and, consequently, no chain. Besides every $E_{i}$ contains a single pseudo-circle of $Y$ denoted by $P_{i}$ henceforth; this vector field does not possess any other pseudo-circle.

It is easily checked that $Y$ is complete with no regular periodic trajectories. On the other hand the set $Y^{-1}(0)$ of singularities of $Y$ consists of $C$ plus the singularities in each $E_{i}$ (a finite number for every $\left.E_{i}\right)$. Since the family $\left\{E_{i}\right\}_{i \in C}$ is locally finite because $\left\{A_{p}\right\}_{p \in C}$ is, it follows that $Y^{-1}(0)$ is discrete and countable. Moreover the set of sources of $Y$ equals $I \cup \tilde{I}$.

Lemma 3.2. There exists a subset $Q \subset M$, which does not exceed dimension $m-1$, such that for every point $q \in(M-Q)$, the $Y$-trajectory of $q$ is regular and included in $M-Q$, its $\alpha$-limit is a source or empty, and its $\omega$-limit a pseudo-circle.

Proof. As the set of zeros of $Y$ is countable and $\bigcup_{i \in I} E_{i}$ can be enclosed in dimension $m-1$, it suffices to consider the points $q$ of $M-\bigcup_{i \in I} E_{i}$ such that $Y(q) \neq 0$. On the other hand since the outset and the inset of any saddle is enclosed in dimension $m-1$, the set of points whose $\alpha$-limit or whose $\omega$-limit is a saddle is enclosed in dimension $m-1$.

Thus it suffices to study those points $q$ in $M-\bigcup_{i \in I} E_{i}$ whose trajectory is regular and intersects some $A_{i}$.

By construction $Y$ is tangent to $E_{i} \equiv\{0\} \times S^{m-1}$ and a limit vector field on this submanifold. Therefore there exists $\{0\} \times \tilde{Q}_{i} \subset E_{i}$, which can be enclosed in dimension $m-2$, such that for any $q \in\left(E_{i}-\{0\} \times \tilde{Q}_{i}\right)$ its $Y$-trajectory has the pseudo-circle $P_{i}$ as $\omega$-limit. Consequently, the pseudo-circle $P_{i}$ is the $\omega$-limit of the $Y$-trajectory of each point of $\left(-\varepsilon_{i}, \varepsilon_{i}\right) \times\left(S^{m-1}-\tilde{Q}_{i}\right)$.

Let $\Phi_{t}$ be the flow of $Y$. The set $\left(-\varepsilon_{i}, \varepsilon_{i}\right) \times \tilde{Q}_{i}$ does not exceed dimension $m-1$ and, since $\mathbb{Q}$ is countable, neither does $\bigcup_{t \in \mathbb{Q}} \Phi_{t}\left(\left(-\varepsilon_{i}, \varepsilon_{i}\right) \times \tilde{Q}_{i}\right)$. In other words, taking into account that $I$ is countable follows that the set of points $q \in\left(M-\bigcup_{j \in I} E_{j}\right)$, with $Y(q) \neq 0$, whose $Y$-trajectory intersects some $A_{i}$ but whose $\omega$-limit is not a pseudo-circle may be enclosed in dimension $m-1$. 
Finally if $M-Q$ is not $Y$-saturated, since all points of the trajectory of $q \in M-Q$ have the same properties, we may replace $Q$ by $Q^{\prime}=M-M^{\prime}$ where $M^{\prime}$ is the $Y$-saturation of $M-Q$.

3.3. Construction of chains. First notice that $Y$ is tangent to $I_{G}=I_{G}^{\max }$ because it is $G$-invariant. Consider a set $Q$ as in Lemma 3.2, which is $Y$-saturated since $M-Q$ is so. On the other hand as $G$ is finite the set $G \cdot Q$ still has the properties of Lemma 3.2. In short, we may suppose that $Q$ is $G$ and $Y$-saturated. Since $\stackrel{\circ}{Q}=\stackrel{\circ}{I}_{G}=\emptyset$ and $I_{G}$ is closed, there exists a countable set $N \subset M-\left(Q \cup I_{G}\right)$ that is dense in $M$ and $G$-saturated. Observe that any orbit of the action of $G$ on $N$ has $\ell$ elements because this action is free. Let $\mathcal{F}$ be the family of all trajectories of $Y$ that intersect $N$. Observe that $\mathcal{F}$ is infinite since no trajectory of $Y$ is locally dense.

Lemma 3.3. Let $U$ be a $G$-invariant vector field on $M, \varphi_{t}$ be its flow and $q$ be a point whose $U$-trajectory is non-periodic. Given $(g, t),(h, s) \in G \times \mathbb{R}$, if $\left(g \circ \varphi_{t}\right)(q)=\left(h \circ \varphi_{s}\right)(q)$ then $t=s$ and either $g=h$ or $q \in I_{G}$.

Proof. From hypotheses, it immediately follows that $\left(\left(h^{-1} g\right) \circ \varphi_{(t-s)}\right)(q)=q$. Therefore

$$
q=\left(\left(h^{-1} g\right) \circ \varphi_{(t-s)}\right)^{\ell}(q)=\left(\left(h^{-1} g\right)^{\ell} \circ \varphi_{\ell(t-s)}\right)(q)=\varphi_{\ell(t-s)}(q)
$$

since $\ell$ is the order of $G$. Hence $\varphi_{\ell(t-s)}(q)=q$, which implies $\ell(t-s)=0$ and $t=s$.

Thus $\left(h^{-1} g\right) \cdot q=q$. If $h^{-1} g \neq e$ then $q \in I_{\left(h^{-1} g\right)} \subset I_{G}$.

Corollary 3.4. The natural action of $G$ on $\mathcal{F}$ is free.

Proof. Assume $g \cdot T=T$ for some $g \in G$ and $T \in \mathcal{F}$. Then given $q \in T$ there exists $t \in \mathbb{R}$ such that $\Phi_{t}(q)=g \cdot q$ and, applying Lemma 3.3 to $Y$ and $q$, it follows that $t=0$ and $g \cdot q=q$. As $q \notin I_{G}$, then $g=e$ must hold.

The set $\mathcal{F}$ is a disjoint union of $G$-orbits, say $\mathcal{F}_{n}, n \geq 3$ (by technical reasons we start at natural three). Let $\mathbb{N}^{\prime}=\mathbb{N}-\{0,1,2\}$. Since by Corollary 3.4 each $\mathcal{F}_{n}$ consists of $\ell$ different trajectories one set $\mathcal{F}=\left\{T_{n k}: n \in \mathbb{N}^{\prime}, k=1, \ldots, \ell\right\}$, where $\mathcal{F}_{n}=\left\{T_{n k}: k=1, \ldots, \ell\right\}$, in such a way that $T_{n k} \neq T_{n^{\prime} k^{\prime}}$ if $(n, k) \neq\left(n^{\prime}, k^{\prime}\right)$. (That is to say first one numbers the $G$-orbits in $\mathcal{F}$ and then, with a second subindex, the elements of every orbit.) 
Consider a sequence of $G$-invariant compact sets $\left\{K_{n}\right\}_{n \in \mathbb{N}}$ such that $K_{n} \subset \stackrel{\circ}{K_{n+1}}$ and $\bigcup_{n \in \mathbb{N}} K_{n}=M$. For every trajectory $T_{n k}$ let $W_{n k}$ be a set of $n-1$ different points of $T_{n k}$ in such a way that:

(a) If $g \cdot T_{n k}=T_{n k^{\prime}}$ then $g \cdot W_{n k}=W_{n k^{\prime}}$.

(b) $W_{n k} \subset M-K_{n}$ if the $\alpha$-limit of $T_{n k}$ is empty.

(c) $W_{n k} \subset \rho_{i}^{-1}\left(B\left(r_{i} / n\right)\right)$ if the $\alpha$-limit of $T_{n k}$ is $i$.

Let $W=\bigcup_{n \in \mathbb{N}^{\prime}, k=1, \ldots, \ell} W_{n k}$; then $C \cup W$ is a countable set whose accumulation points are the minima and the maxima of $\mu$, i.e. the elements of $I \cup \tilde{I}$. Therefore $C \cup W$ is closed and there exists a function $\tau: M \rightarrow[0,1] \subset \mathbb{R}$ such that $\tau^{-1}(0)=C \cup W$. Set $X=\tau Y$. It easily seen that:

(1) $X^{-1}(0)=Y^{-1}(0) \cup W$ is countable and closed. The set of its accumulation points equals $I \cup \tilde{I}$.

(2) $X$ is complete and has no periodic regular trajectory.

(3) $\left\{P_{i}\right\}_{i \in I}$ is the family of all pseudo-circles of $X$.

(4) Let $C_{n k}$ be the family of $X$-trajectories of $T_{n k}-W_{n k}$ endowed with the order induced by that of $T_{n k}$ as $Y$-trajectory. Then $C_{n k}$ is a chain of $X$ of order $n$ whose rivets are the points of $W_{n k}$. Besides $C_{n 1}, \ldots, C_{n \ell}$ are the only chains of $X$ of order $n$ and hence $\left\{C_{n k}\right\}, k=1, \ldots, \ell, n \in \mathbb{N}^{\prime}$, is the set of all the chains of $X$.

Denote by $H_{n k}$ the last link of $C_{n k}$ and by $P_{\lambda(n, k)}$ the $\omega$-limit of $H_{n k}$ (therefore $\lambda$ is a map from $\mathbb{N}^{\prime} \times\{1, \ldots, \ell\}$ to $\left.I\right)$.

(5) $\bigcup_{n \in \mathbb{N}^{\prime}, k=1, \ldots, \ell} H_{n k}$ is dense in $M$.

Remark 3.5. Notice that the chains $C_{n k}$ given by (4) can be described in topological terms as finite sequences of $X$-trajectories such that:

(i) Between two consecutive links, the $\omega$-limit of the first one equals the $\alpha$-limit of the second one. Moreover this set consists in a topological rivet.

(ii) The $\alpha$-limit of the first link is an accumulation point of $X^{-1}(0)$ or empty.

(iii) The $\omega$-limit of the last link is a pseudo-circle.

Observe that $\left\{C_{n k}\right\}, k=1, \ldots, \ell, n \in \mathbb{N}^{\prime}$, is the set of all the objects satisfying (i), (ii) and (iii) above because $W$ is the set of topological rivets of $X$ ( $Y$ has no rivet). Therefore any continuous automorphisms of $X$ maps chains to chains. 
By definition the roll $R_{i}, i \in I$, is the union of all $H_{n k}$ whose $\omega$-limit equals $P_{i}$.

3.4. $X$ is a suitable vector field. In this subsection $\Phi_{t}$ will be the flow of $X$. Consider a homeomorphism $f: M \rightarrow M$ such that $f \circ \Phi_{t}=\Phi_{t} \circ f$ for any $t \in \mathbb{R}$.

Proposition 3.6. For each roll $R_{i}$ there exist $t_{i} \in \mathbb{R}$ and $g_{i} \in G$ such that $f=g_{i} \circ \Phi_{t_{i}}$ on $R_{i}$.

Proof. Fixed a $R_{i}$ consider a chain $C_{n k}$ whose last link $H_{n k}$ has $P_{i}$ as $\omega$-limit. By Remark 3.5, $f\left(C_{n k}\right)$ is a chain of of order $n$, so $f\left(C_{n k}\right)=C_{n k^{\prime}}$ and $f\left(H_{n k}\right)=H_{n k^{\prime}}$ for some $k^{\prime} \in\{1, \ldots, \ell\}$. Moreover $f\left(P_{i}\right)$ is the $\omega$-limit of $H_{n k^{\prime}}$.

As $\mathcal{F}_{n}=\left\{T_{n 1}, \ldots, T_{n \ell}\right\}$ is an orbit of the action of $G$ on $\mathcal{F}$, there exists $h \in G$ such that $h \cdot T_{n k}=T_{n k^{\prime}}$, hence $h \cdot H_{n k}=H_{n k^{\prime}}$. Now by composing $f$ on the left with $h^{-1}$ we may assume $f\left(C_{n k}\right)=C_{n k}, f\left(H_{n k}\right)=H_{n k}$ and $f\left(P_{i}\right)=P_{i}$.

Since $f$ commutes with any $\Phi_{t}$ and the regular trajectory of $P_{i}$ is not periodic, there exists a single $t_{i} \in \mathbb{R}$ such that $f=\Phi_{t_{i}}$ on $P_{i}$.

Now consider any $H_{a b},(a, b) \in \mathbb{N}^{\prime} \times\{1, \ldots, \ell\}$, with $\omega$-limit $P_{i}$. Then $f\left(H_{a b}\right)$, which is the last link of $f\left(C_{a b}\right)$, has $P_{i}$ as $\omega$-limit too.

Recall that the $G$-orbit of $i$ possesses $\ell$ elements, that is to say if $i \notin I_{G}$. Therefore there is a single $H_{a b^{\prime}}$ with $\omega$-limit $P_{i} ; H_{a b}$ itself. Indeed, there are only $\ell$ chains of order $a$ and the $\omega$-limits of their last links are included in the disjoint union $\bigcup_{g \in G}\left(g \cdot A_{i}\right)$. In other words $f\left(H_{a b}\right)=H_{a b}$.

But $H_{a b}$ is a non-periodic regular trajectory and $f$ commutes with the flow $\Phi_{t}$, so there exists $t^{\prime} \in \mathbb{R}$ such that $f=\Phi_{t^{\prime}}$ on $H_{a b}$. As $P_{i}$ is the $\omega$-limit of $H_{a b}$ one has $f=\Phi_{t^{\prime}}$ on $P_{i}$ too, hence $t^{\prime}=t_{i}$.

From Proposition 3.6, it immediately follows:

Corollary 3.7. For every roll $R_{i}$ there exist $t_{i} \in \mathbb{R}$ and $g_{i} \in G$ such that $f=g_{i} \circ \Phi_{t_{i}}$ on $\bar{R}_{i}$.

Lemma 3.8. The family $\left\{\bar{R}_{i}\right\}_{i \in I}$ is locally finite and $\bigcup_{i \in I} \bar{R}_{i}=M$.

Proof. For the first part it suffices to show that $\left\{R_{i}\right\}_{i \in I}$ is locally finite. From the fact that $P_{i}$ is included in $A_{i}$ follows that $\mu\left(R_{i}\right)$ is low bounded by $\mu(i)$. But $I$ is a discrete set and $\mu$ a non-negative proper Morse function, so in every compact set $\mu^{-1}((-\infty, a])$ there are only 
a finite number of elements of $I$. Therefore $\mu^{-1}((-\infty, a])$ and of course $\mu^{-1}(-\infty, a)$ only intersect a finite number of rolls $R_{i}$. Finally, observe that $M=\bigcup_{a \in \mathbb{R}} \mu^{-1}(-\infty, a)$.

By construction of $X$ (see Property (5)) $\bigcup_{n \in \mathbb{N}^{\prime}, k=1, \ldots, \ell} H_{n k}$ is dense in $M$. On the other hand $\bigcup_{i \in I} \bar{R}_{i}$ is closed because $\left\{\bar{R}_{i}\right\}_{i \in I}$ is locally finite, so $\bigcup_{i \in I} \bar{R}_{i}$ is a closed set that includes $\bigcup_{n \in \mathbb{N}^{\prime}, k=1, \ldots, \ell} H_{n k}$.

Lemma 3.9. All scalars $t_{i}$ given by Corollary 3.7 are equal.

Proof. Assume that the family $\left\{t_{i}\right\}_{i \in I}$ possesses two or more elements. Fixed one of them, say $t$, set $D_{1}$ the union of all $\bar{R}_{i}$ such that $t_{i}=t$ and $D_{2}$ the union of all $\bar{R}_{i}$ such that $t_{i} \neq t$. By Lemma 3.8, $D_{1}$ and $D_{2}$ are closed and $M=D_{1} \cup D_{2}$.

On the other hand if $p \in D_{1} \cap D_{2}$ then there exist $\bar{R}_{i} \subset D_{1}$ and $\bar{R}_{j} \subset D_{2}$ such that $p \in \bar{R}_{i} \cap \bar{R}_{j}$; so $f(p)=\left(g_{i} \circ \Phi_{t_{i}}\right)(p)=\left(g_{j} \circ \Phi_{t_{j}}\right)(p)$. As $t_{i} \neq t_{j}$ from Lemma 3.3 applied to $X$ and $p$ follows that the $X$-orbit of $p$ is periodic. Hence $X(p)=0$ since $X$ has no periodic regular trajectories, which implies that $D_{1} \cap D_{2}$ is countable. Consequently $M-D_{1} \cap D_{2}$ is connected. But $M-D_{1} \cap D_{2}=\left(D_{1}-D_{1} \cap D_{2}\right) \cup\left(D_{2}-D_{1} \cap D_{2}\right)$ where the terms of this union are non-empty, disjoint and closed in $M-D_{1} \cap D_{2}$, contradiction.

Now composing $f$ with $\Phi_{-t}$ where $t$ is the scalar given by Corollary 3.7 and Lemma 3.9 we may assume, without lost of generality, that $f(x)=g_{x} \cdot x$ for any $x \in M$ where $g_{x} \in G$. For finishing the proof of the existence of $(g, t) \in G \times \mathbb{R}$ such that $f=g \circ \Phi_{t}$ it suffices to apply the following result:

Lemma 3.10. Consider a continuous and injective map $\tau: M \rightarrow M$. Assume for every $x \in M$ there exists $g_{x} \in G$ such that $\tau(x)=g_{x} \cdot x$. Then $\tau=g$ for some $g \in G$.

Proof. Given $g \in G$ set $D_{g}:=\left\{p \in M-I_{G}: \tau(p)=g \cdot p\right\}$. As the space is Hausdorff $G_{g}$ is closed in $M-I_{G}$. Moreover $D_{g} \cap D_{h}=\emptyset$ when $g \neq h$ since the action of $G$ on $M-I_{G}$ is free. As $G$ is finite and $M-I_{G}=\bigcup_{h \in G} D_{h}$, every $D_{g}$ is open too so union of connected components of $M-I_{G}$.

Some of the sets $D_{g}, g \in G$, has to be non-empty and by composing $\tau$ on the left with a suitable element of $G$ one may assume $D_{e} \neq \emptyset$. Let us see that $\bar{D}_{e}$ is open in $M$. If so the proof is finished since $M$ is connected and necessarily $\bar{D}_{e}=M$.

Consider any $p \in \bar{D}_{e}$. If $p \in M-I_{G}$ then $p \in D_{e}$ and it is an interior point. Now assume $p \in I_{h}=I_{h}^{\max }$ for some $h \in G-\{e\}$. Then around $p$ there exist coordinates $\left(x_{1}, \ldots, x_{m}\right)$ 
whose domain $D$ is diffeomorphic through these coordinates to an open ball $B(r)$ such that $p \equiv 0$ and $h$ is given by the symmetry $\Gamma(x)=\left(x_{1}, \ldots, x_{m-1},-x_{m}\right)$.

Let $S^{+}$be the open half domain defined by $x_{m}>0$ and $S^{-}$that given by $x_{m}<0$. If $r$ is sufficiently small then $S^{+} \cup S^{-} \subset M-I_{G}$, and $I_{G} \cap D$ and $I_{h} \cap D$ are equal and defined by $x_{m}=0$ (see (a) of Lemma 3.1). As $p \in \bar{D}_{e}$ and $D_{e}$ is an union of connected components of $M-I_{G}$ necessarily one at least of the foregoing open half domains is included in $D_{e}$. Assume $S^{+} \subset D_{e}$, the other case is similar. If $S^{-} \not \subset D_{e}$ then $S^{-} \cap D_{e}=\emptyset$ and there is some $\bar{g} \in G-\{e\}$ such that $S^{-} \subset D_{\bar{g}}$ because $S^{-}$is connected.

By continuity $\bar{g} \cdot p=\tau(p)$ and $e \cdot p=\tau(p)$, hence $\bar{g} \cdot p=p$ and by (a) of Lemma 3.1 one has $\bar{g}=h$. Therefore $\tau$ on $S^{-}$equals $h$. Thus $\tau(0, \ldots, 0,-\varepsilon)=(0, \ldots, 0, \varepsilon)=e \cdot(0, \ldots, 0, \varepsilon)=$ $\tau(0, \ldots, 0, \varepsilon), \varepsilon>0$, and $\tau$ is not injective, contradiction. In short $S^{-} \subset D_{e}$ and necessarily $p \in D \subset \bar{D}_{e}$.

Finally, if $g \circ \Phi_{t}=I d_{M}$ then $I d_{M}=\left(g \circ \Phi_{t}\right)^{\ell}=g^{\ell} \circ \Phi_{\ell t}=\Phi_{\ell t}$. As $X$ has regular nonperiodic trajectories $t=0$, so $g=e$. This fact implies the injectivity of the morphism from $G \times \mathbb{R}$ to $\operatorname{Aut}_{0}(X)$. Therefore the main theorem is proved under the supplementary hypothesis $I_{G}=I_{G}^{\max }$.

Remark 3.11. Consider a function $\varphi: M \rightarrow \mathbb{R}$ that is $G$-invariant, positive and bounded. Then $\varphi X$ is a complete vector field. Besides $X$ and $\varphi X$ have the same trajectories (with different speeds but the same orientation by the time), $\alpha$ and $\omega$-limits, pseudo-circles, rolls, rivets and chains. Therefore reasoning as before but this time with $\varphi X$ shows that $(g, t) \in$ $G \times \mathbb{R} \rightarrow g \circ \tilde{\Phi}_{t} \in \operatorname{Aut}_{0}(\varphi X)$ is a group isomorphism where $\tilde{\Phi}_{t}$ is the flow of $\varphi X$.

In other words $\varphi X$ is a suitable vector field too.

\section{The GEneral CASE}

In this section the main result will be proved in the general case by reducing it to the almost free one.

Given $g \in G-\{e\}$ let $J_{g}$ be the set of those points $p \in I_{g}$ such that the dimension of $I_{g}$ at $p$ is $\leq m-2$. Set $J_{G}:=\bigcup_{g \in G-\{e\}} J_{g}$. It is easily seen that $J_{G}$ is a $G$-invariant closed set and $M-J_{G}$ a $G$-invariant dense open set. 
One will say that the dimension of a point $p \in J_{G}$ is zero if the dimension at $p$ of every $I_{g}$ such that $p \in J_{g}$, is zero. Let $S_{0}$ be the set of all points of $J_{G}$ of dimension zero. Clearly $S_{0}$ is $G$-invariant and $S_{0} \subset J_{G}$.

By making use of normal coordinates with respect to a $G$-invariant Riemannian metric, centered at points of $J_{G}$, it is easily checked that:

(1) $S_{0}$ has no accumulation point so it is countable and closed.

(2) Every $q \in S_{0}$ possesses a neighborhood whose intersection with $J_{G}$ equals $\{q\}$.

(3) For any $q \in J_{G}-S_{0}$ and any neighborhood $A$ of $q$ the set $A \cap J_{G}$ is uncountable.

Since $M$ is paracompact (even more $\sigma$-compact) from (1) and (2) follows the existence of a locally finite family of disjoint open sets $\tilde{\mathcal{A}}=\left\{\tilde{A}_{q}\right\}_{q \in S_{0}}$ such that every $\tilde{A}_{q} \cap S_{0}=\{q\}$. As $S_{0}$ is $G$-invariant and $G$ is finite shrinking the elements of $\tilde{\mathcal{A}}$ allows us to assume that this family is $G$-invariant. Even more one can suppose that each $\tilde{A}_{q}$ is a domain of normal coordinates centered at $q$ of some $G$-invariant Riemannian metric.

For every $q \in S_{0}$ consider a set $q \in C_{q} \subset \tilde{A}_{q}$ that in the normal coordinates mentioned before is a closed non-trivial segment sufficiently small. Set $D_{q}:=\left\{(g, p) \in G \times S_{0}: g \cdot p=q\right\}$ and $E_{q}:=\bigcup_{(g, p) \in D_{q}} g \cdot C_{p}$. Then $E_{q} \subset \tilde{A}_{q}$ so the family $\left\{E_{q}\right\}_{q \in S_{0}}$ is locally finite, hence $S_{1}:=\bigcup_{q \in S_{0}} E_{q}$ is closed. Moreover $S_{1}$ is $G$-invariant and any neighborhood of any point of $S_{1}$ includes uncountably many points of $S_{1}$. On the other hand we may assumed that $M-S_{1}$ is connected without loss of generality.

Thus the set $\tilde{M}:=M-\left(J_{G} \cup S_{1}\right)$ is $G$-invariant, connected, dense and open, since $S_{1}$ can be enclosed in dimension one and $J_{g}$ in dimension $m-2$ and $M-S_{1}$ was connected. Besides each neighborhood of every point of $J_{G} \cup S_{1}$ contains uncountably many elements of $J_{G} \cup S_{1}$.

On the other hand the action of $G$ on $\tilde{M}$ is almost free. Indeed, if $q \in I_{g} \cap \tilde{M}$ for some $g \in G-\{e\}$ then $q \notin J_{g}$ so the dimension of $I_{g}$ at $q$ equals $m-1$ and $q \in I_{g}^{\max }$.

By [8, Proposition 5.5] there exists a bounded function $\varphi: M \rightarrow \mathbb{R}$, which is positive on $\tilde{M}$ and vanishes on $M-\tilde{M}$, such that the vector field $\hat{X}$ on $M$ defined by $\hat{X}=\varphi X$ on $\tilde{M}$ and $\hat{X}=0$ on $M-\tilde{M}$ is differentiable.

Notice that $g_{*}^{-1}(\hat{X})$ equals $(\varphi \circ g) X$ and zero on $M-\tilde{M}$. Therefore by taking $\ell^{-1} \sum_{g \in G}(\varphi \circ g)$ instead of $\varphi$ we my assume that $\varphi$ is $G$-invariant, which implies that $\hat{X}$ is $G$-invariant too.

Given a singularity $p$ of $\hat{X}$ one has two possibilities: 
(a) Any neighborhood of $p$ contains uncountably many zeros of $\hat{X}$; that is to say $p \in$ $M-\tilde{M}$.

(b) There is a neighborhood of $p$ that only includes countably many zeros of $\hat{X}$; that is to say $p \in \tilde{M}$ and $X(p)=0$.

Consider $f \in \operatorname{Aut}_{0}(\hat{X})$. From (a) and (b) follows that $f(M-\tilde{M})=M-\tilde{M}$ and $f(\tilde{M})=\tilde{M}$. Thus the restriction of $f$ to $\tilde{M}$ is a continuous automorphism of $\hat{X}_{\mid M}=\varphi X$ and, by Section 3. there exist $g \in G$ and $t \in \mathbb{R}$ such that $f=g \circ \hat{\Phi}_{t}$ on $\tilde{M}$ where $\hat{\Phi}_{t}$ is the flow of $\hat{X}$. By continuity $f=g \circ \hat{\Phi}_{t}$ everywhere. The uniqueness of $g$ and $t$ is obvious. In short the main result is proved in the general case.

\section{ACtions on manifolds With BOUndary}

Let $P$ be a $m$-manifold with nonempty boundary $\partial P$. Then each homeomorphism $f: P \rightarrow$ $P$ induces a homeomorphism $f: \partial P \rightarrow \partial P$. Therefore the same reasoning as in Section 4 of [8] shows that the main result of the present paper also holds for a connected manifold $P$, of dimension $m \geq 2$, with nonempty boundary and a finite subgroup $G$ of $\operatorname{Diff}(M)$.

\section{EXAMPLES}

Example 6.1. On $\mathbb{R}^{2}$ consider the group of two elements $G=\{e, g\}$ where $g(x)=\left(-x_{1}, x_{2}\right)$. Then the action of $G$ on $\mathbb{R}^{2}$ is almost free and $I_{G}^{\max }=\{0\} \times \mathbb{R}$. For constructing a suitable vector field $X$ as in Section 3 , one can start with the Morse function $\mu=\left(x_{1}^{2}-1\right)^{2}+x_{2}^{2}$ that has two minima at $(1,0)$ and $(-1,0)$ respectively and a saddle at the origin.

Therefore at the end of the process $X$ has two pseudo-circles around $(1,0)$ and $(-1,0)$ respectively. Moreover the set of singularities of $X$ is countable and accumulates towards $(1,0),(-1,0)$ and the infinity. Observe that $I_{G}^{\max }$ consists of a singular point and two regular trajectories with the singular point as $\alpha$-limit and empty $\omega$-limit.

In a similar way, on $S^{2}$ one may consider the group $G=\{e, g\}$ where now $g(x)=$ $\left(x_{1}, x_{2},-x_{3}\right)$ and the Morse function $\mu=2 x_{1}^{2}+x_{2}^{2}$ that has two minima at $(0,0, \pm 1)$, two maxima at $( \pm 1,0,0)$ and two saddles at $(0, \pm 1,0)$. The action of $G$ is almost free and there is no minimum on $I_{G}^{\max }=S^{2} \cap\left(\mathbb{R}^{2} \times\{0\}\right)$.

Example 6.2. Let $M$ be a connected compact manifold of dimension $m \geq 2$. Given $G$, a finite group of diffeomorphisms of $M$, and a $G$-invariant Morse function $\mu$, let $X$ be the 
gradient vector field of $\mu$ with respect to a $G$-invariant Riemannian metric. Then, although the group of smooth automorphisms of $X$, namely $\operatorname{Aut}(X)$, may equal $G \times \mathbb{R}$ (e.g. in $[8$, Example 5.2]), the group $\operatorname{Aut}_{0}(X)$ is strictly greater than $G \times \mathbb{R}$.

Indeed, first note that there always exist a minimum and a maximum of $\mu$, that we denote by $p$ and $q$, and a trajectory $\gamma$ of $X$ whose $\alpha$-limit and $\omega$-limit are $p$ and $q$ respectively. Consider a closed sufficiently small $(m-1)$-disk $D$ transverse to $X$ and intersecting $\gamma$ just once. We may suppose, without lost of generality, that every trajectory of $X$ intersects $D$ at most once and if so its $\alpha$-limit equals $p$ and its $\omega$-limit $q$.

Let $E$ be the set of those points of $M$ whose trajectory meets $D$. Then $E$ is diffeomorphic to $D \times \mathbb{R}$ in such a way that $X$ becomes $\partial / \partial s$ where $D \times \mathbb{R}$ is endowed with coordinates $(x, s)=\left(x_{1}, \ldots, x_{m-1}, s\right)$.

For each continuous function $\lambda: D \rightarrow \mathbb{R}$ such that $\lambda(\partial D)=0$ one defines $f \in \operatorname{Aut}_{0}(X)$ to be $f=I d$ on $M-E$ and $f(x, s)=\Phi_{\lambda(x)}(x, s)=(x, s+\lambda(x))$ on $E$, where $\Phi_{t}$ is the flow of $X$. As $\bar{E}-E=((\partial D) \times \mathbb{R}) \cup\{p, q\}$ our $f$ is continuous. Its inverse is given by $-\lambda$ and obviously $f$ is an automorphism of $X$, which in general does not belong to $G \times \mathbb{R}$.

Other way for constructing such a $f$ is to consider a homeomorphism $\tau: D \rightarrow D$ with $\tau_{\mid \partial D}=I d_{\mid \partial D}$ and set $f(x, s)=(\tau(x), s)$ on $E, f=I d$ elsewhere.

Observe that an analogous construction can be done if the gradient field is slightly modified, namely if one adds a finite number of new singularities of index zero. Thus, in general, the group of continuous automorphisms of vector fields constructed in [8] is strictly greater than $G \times \mathbb{R}$ (for the non-compact case the reasoning above can be easily adapted if there is at least a maximum). In other words, these vector fields determine $G$ in the smooth category but not in the continuous one.

The next two examples are extensions of Corollary [1.2 to situations where differentiability at every point is not assured. One has:

Proposition 6.3. Consider a finite group $G$ of homeomorphisms of a connected $C^{\infty}$ manifold $M$ of dimension $\geq 2$. Let $A$ be a $G$-invariant open set of $M$. Assume that:

(a) A is connected and dense, and every neighborhood of each point of $M-A$ contains uncountably many points of $M-A$.

(b) Under restriction each element of $G$ is a diffeomorphism of $A$. 
Then there exists a $G$-invariant smooth, and therefore continuous, flow $\psi: \mathbb{R} \times M \rightarrow M$ such that the map

$$
\begin{aligned}
G \times \mathbb{R} & \rightarrow \operatorname{Aut}_{0}(\psi) \\
(g, t) & \mapsto g \circ \psi_{t}
\end{aligned}
$$

is a group isomorphism.

Proof. Consider $G$ under restriction as a group of diffeomorphisms of $A$ and define $J_{G}$ and $S_{1}$ as in Section 4 (for $A$ of course). Set $\tilde{M}=M-\left(J_{G} \cup S_{1} \cup(M-A)\right)=A-J_{G} \cup S_{1}$. Then the action of $G$ on $\tilde{M}$ is almost free, which gives rise to a suitable vector field $X$ on $\tilde{M}$.

Since $J_{G} \cup S_{1} \cup(M-A)$ is a closed set of $M$ with empty interior, a vector field $\hat{X}$ on $M$ that

(1) vanishes in $J_{G} \cup S_{1} \cup(M-A)$ and

(2) on $\tilde{M}$ equals $\varphi X$ for a suitable function $\varphi: M \rightarrow \mathbb{R}$,

can be constructed as in Section 4 ,

The flow of $\hat{X}$ has the required properties. Indeed, any neighborhood of any point of $J_{G} \cup S_{1} \cup(M-A)$ includes uncountably many points of this set, and one can reason as in the second part of Section 4 .

Example 6.4. Following the notation in [4, pp. 23-24], in $\mathbb{C}^{4}$ endowed with coordinates $z=\left(z_{0}, z_{1}, z_{2}, z_{3}\right)$ the equations

$$
\begin{gathered}
z_{0}^{3}+z_{1}^{2}+z_{2}^{2}+z_{3}^{2}=0 \\
\sum_{k=0}^{3} z_{k} \bar{z}_{k}=1
\end{gathered}
$$

define a smooth real submanifold that is diffeomorphic to the standard sphere $S^{5}$ such that $\beta(z)=\left(e^{2 \pi i / 3} z_{0}, z_{1}, z_{2}, z_{3}\right)$ defines a diffeomorphism of $S^{5}$ of order three, whose set of fixed points is (diffeomorphic to) $\mathbb{R} P^{3}$ [4, REMARKS p. 24].

The (topological) suspension of $S^{5}$ and that of $\beta$ give rise to a homeomorphism $f: S^{6} \rightarrow S^{6}$ of order three, whose set of fixed points is (homeomorphic to) the suspension of $\mathbb{R} P^{3}$ in such a way that the vertices are the poles. Therefore $f$ cannot be smoothed otherwise the suspension of $\mathbb{R} P^{3}$ has to be a differentiable manifold, which is not the case.

Let $G$ be the group of homeomorphisms of $S^{6}$ spanned by $f$, whose order equals three. Clearly $G$ cannot be smoothed. However, away of the poles $G$ is a group of diffeomorphisms. 
Consider a meridian (that is the intersection of $S^{6} \subset \mathbb{R}^{7}$ with a plane passing through the origin and the poles) and saturate it under the action of $G$ for constructing a $G$-invariant compact set $C$. Finally set $A=S^{6}-C$ and apply Proposition 6.3 for concluding that, even if $G$ cannot be smoothed, there exists a differentiable flow on $S^{6}$ that determines $G$.

Theorem 6.5. Let $G$ be a finite group of homeomorphisms of a connected compact topological 4-manifold $M$ with no boundary. Assume that the action of $G$ is free. Then there exist a continuous flow $\widetilde{\Phi}$ that determines $G$.

We devote the rest of this section to the proof of Theorem 6.5.

Let $P=M / G$ be the topological quotient manifold and $\pi: M \rightarrow P$ the canonical projection, which is a covering.

Fix a point $a$ of $P$. Then $P^{\prime}:=P-\{a\}$ has a structure of smooth manifold (Quinn [7]). The pull-back of this structure defines a smooth structure in $M^{\prime}:=M-\pi^{-1}(a)=\pi^{-1}\left(P^{\prime}\right)$ in such a way that the natural action of $G$ on $M^{\prime}$ is smooth and $\pi: M^{\prime} \rightarrow P^{\prime}$ is a smooth covering.

Consider a set $a \in C \subset P$ that with respect to some topological coordinates centered at $a$ is a closed non-trivial segment sufficiently small; assume that $a$ is one of its vertices. Then $\pi^{-1}(C)$ is a disjoint union of compact sets $C_{1} \cup \ldots \cup C_{\ell}$ where $\ell$ is the order of $G$ and each $\pi: C_{j} \rightarrow C$ a homeomorphism. Notice that $P-C$ is connected and dense in $P$, and $M-\pi^{-1}(C)$ is connected, dense in $M$ and $G$-invariant.

Now construct a suitable vector field $X$ on $M-\pi^{-1}(C)$. Since $\pi^{-1}(C-\{a\})$ is closed in $M^{\prime}$, a vector field $\widehat{X}$ that vanishes on $\pi^{-1}(C-\{a\})$ and equals $\varphi X$ on $M-\pi^{-1}(C)$ for a suitable function $\varphi: M^{\prime} \rightarrow \mathbb{R}$ can be constructed as in Section 4 .

On the other hand the flow $\widehat{\Phi}$ of $\widehat{X}$ can be extended into a continuous flow $\widetilde{\Phi}: \mathbb{R} \times M \rightarrow M$ by setting $\widetilde{\Phi}(\mathbb{R} \times\{b\})=b$ for every $b \in \pi^{-1}(a)$. Accept this fact by the moment; we will prove it later on. If $f: M \rightarrow M$ is a continuous automorphism of $\widetilde{\Phi}$, then $f\left(\pi^{-1}(C)\right)=$ $\pi^{-1}(C)$ by the same reason as in Section 4 (replace singularities of $\widehat{X}$ by stationary points of the flow $\widetilde{\Phi}$ and take into account that any neighborhood of any point of $\pi^{-1}(C)$ contains uncountably many points of $\pi^{-1}(C)$ ). Therefore $f: M-\pi^{-1}(C) \rightarrow M-\pi^{-1}(C)$ is a continuous automorphism of $\widehat{X}$ and hence $f=g \circ \widehat{\Phi}_{t}=g \circ \widetilde{\Phi}_{t}$ on $M-\pi^{-1}(C)$ for some $g \in G$ and $t \in \mathbb{R}$. Since $M-\pi^{-1}(C)$ is dense in $M$, by continuity $f=g \circ \widetilde{\Phi}_{t}$ everywhere.

Clearly if $g \circ \widetilde{\Phi}_{t}=I d$ then $g=e$ and $t=0$. 
In short, even if $M$ has no smooth structure, the continuous flow $\widetilde{\Phi}$ determines $G$.

Let us prove that $\widetilde{\Phi}$ is a continuous flow. The only difficult point is the continuity. Note that as $\widehat{\Phi}$ is $G$-invariant there is a smooth flow $\psi: \mathbb{R} \times P^{\prime} \rightarrow P^{\prime}$ such that $\pi \circ \widehat{\Phi}=\psi \circ(I d \times \pi)$ (it is the flow associated to the projection of $\widehat{X}$ onto $P^{\prime}$ ). Denote by $\widetilde{\psi}: \mathbb{R} \times P \rightarrow P$ the extension of $\psi$ defined by setting $\widetilde{\psi}(\mathbb{R} \times\{a\})=a$. Observe that $\pi \circ \widetilde{\Phi}=\widetilde{\psi} \circ(I d \times \pi)$.

Lemma 6.6. $\widetilde{\psi}$ is continuous.

Proof. As before the difficult point is the continuity. For checking it one will show that $\widetilde{\psi}:[a, b] \times P \rightarrow P$ is continuous for any $a<b$ belonging to $\mathbb{R}$.

Consider a map $s: E_{1} \rightarrow E_{2}$ between locally compact but not compact topological spaces. Denote by $\mathcal{A}\left(E_{k}\right), k=1,2$, the Alexandroff compactification of $E_{k}$ and by $\mathcal{A}(s): \mathcal{A}\left(E_{1}\right) \rightarrow$ $\mathcal{A}\left(E_{2}\right)$ the extension of $s$ that maps the infinity point of $\mathcal{A}\left(E_{1}\right)$ to that of $\mathcal{A}\left(E_{2}\right)$. Recall that $\mathcal{A}(s)$ is continuous if and only if $s$ is proper.

The map $h:[a, b] \times P^{\prime} \rightarrow[a, b] \times P^{\prime}$ given by $h(t, x)=(t, \psi(t, x))$ is a homeomorphism and hence $\mathcal{A}(h): \mathcal{A}\left([a, b] \times P^{\prime}\right) \rightarrow \mathcal{A}\left([a, b] \times P^{\prime}\right)$ is continuous.

In turn the second projection $\pi_{2}:[a, b] \times P^{\prime} \rightarrow P^{\prime}$ is proper, so $\mathcal{A}\left(\pi_{2}\right): \mathcal{A}\left([a, b] \times P^{\prime}\right) \rightarrow \mathcal{A}\left(P^{\prime}\right)$ is continuous.

Since the Alexandroff compactification is the smallest one among the Hausdorff compactifications, the map $g:[a, b] \times \mathcal{A}\left(P^{\prime}\right) \rightarrow \mathcal{A}\left([a, b] \times P^{\prime}\right)$ that equals the identity on $[a, b] \times P^{\prime}$ and maps $[a, b] \times\{\infty\}$ to $\infty$ is continuous.

Finally if one identifies $P$ to $\mathcal{A}\left(P^{\prime}\right)$ by regarding a like the infinity point, then $\widetilde{\psi}=\mathcal{A}\left(\pi_{2}\right) \circ$ $\mathcal{A}(h) \circ g$.

Corollary 6.7. $\widetilde{\Phi}$ is continuous.

Proof. Consider the map $l: \mathbb{R} \times M \rightarrow P$ given by $l(t, x)=\widetilde{\psi}(t, \pi(x))$ and a point $v \in M^{\prime}$. As $\mathbb{R}$ is contractile, then $l$ is homotopic to the map

$$
\begin{aligned}
\mathbb{R} \times M & \rightarrow P \\
(t, x) & \mapsto \pi(x)
\end{aligned}
$$

Therefore $l_{\sharp}\left(\pi_{1}(\mathbb{R} \times M,(0, v))\right)=\pi_{\sharp}\left(\pi_{1}(M, v)\right)$ and hence, with respect to the covering $\pi: M \rightarrow P$, there exists a lift $L: \mathbb{R} \times M \rightarrow M$ of $l$, with initial condition $L(0, v)=v$. Moreover $L=\widetilde{\Phi}$ on $\mathbb{R} \times M^{\prime}$ since one knows that $\widetilde{\Phi}$ is continuous on $\mathbb{R} \times M^{\prime}$ and $\pi \circ \widetilde{\Phi}=\widetilde{\psi} \circ(I d \times \pi)$. 
Finally as $\widehat{X}$ vanishes on $\pi^{-1}(C-\{a\})$, it follows that $L=\widetilde{\Phi}=I d$ on $\mathbb{R} \times \pi^{-1}(C-\{a\})$. By continuity $L(\mathbb{R} \times\{b\})=b$ for each $b \in \pi^{-1}(a)$. Thus $L=\widetilde{\Phi}$ everywhere.

\section{Appendix}

In this section $B(r), r>0$, will be the ball in $\mathbb{R}^{m}$, endowed with coordinates $\left(x_{1}, \ldots, x_{m}\right)$, of center the origin and radius $r$, and $\Gamma: \mathbb{R}^{m} \rightarrow \mathbb{R}^{m}$ the symmetry given by $\Gamma\left(x_{1}, \ldots, x_{m}\right)=$ $\left(x_{1}, \ldots, x_{m-1},-x_{m}\right)$.

Lemma 7.1. Consider a function $\mu$ defined around the origin of $\mathbb{R}^{m}$ and $\Gamma$-invariant. Assume that the origin is a non-degenerated singularity. Then about the origin there exist coordinates $\left(y_{1}, \ldots, y_{m}\right)$ such the coordinates of the origin are still $(0, \ldots, 0)$,

$$
\mu=\sum_{j=1}^{k} y_{j}^{2}-\sum_{j=k+1}^{m-1} y_{j}^{2}+\varepsilon y_{m}^{2}+\mu(0)
$$

where $\varepsilon= \pm 1$, and $\Gamma\left(y_{1}, \ldots, y_{m}\right)=\left(y_{1}, \ldots, y_{m-1},-y_{m}\right)$.

Proof. As $\mu$ is $\Gamma$-invariant its restriction to the hyperplane $H$ defined by $x_{m}=0$ has a nondegenerated singularity at the origin. Therefore coordinates $\left(x_{1}, \ldots, x_{m}\right)$ can be replaced by coordinates $\left(y_{1}, \ldots, y_{m-1}, x_{m}\right)$ in such a way that $\Gamma\left(y_{1}, \ldots, y_{m-1}, x_{m}\right)=\left(y_{1}, \ldots, y_{m-1},-x_{m}\right)$ and

$$
\mu\left(y_{1}, \ldots, y_{m-1}, 0\right)=\sum_{j=1}^{k} y_{j}^{2}-\sum_{j=k+1}^{m-1} y_{j}^{2}+\mu(0) .
$$

On the other hand from the Taylor expansion in variable $x_{m}$ transversely to $H$ it follows

$$
\begin{aligned}
\mu\left(y_{1}, \ldots, y_{m-1}, x_{m}\right) & =\mu\left(y_{1}, \ldots, y_{m-1}, 0\right) \\
& +x_{m} \frac{\partial \mu}{\partial x_{m}}\left(y_{1}, \ldots, y_{m-1}, 0\right)+x_{m}^{2} f\left(y_{1}, \ldots, y_{m-1}, x_{m}\right) .
\end{aligned}
$$

By the $\Gamma$-invariance $\frac{\partial \mu}{\partial x_{m}}\left(y_{1}, \ldots, y_{m-1}, 0\right)=0$ and $f\left(y_{1}, \ldots, y_{m-1},-x_{m}\right)=f\left(y_{1}, \ldots, y_{m-1}, x_{m}\right)$. Moreover $2 f(0)=\frac{\partial^{2} \mu}{\partial x_{m}^{2}}(0) \neq 0$ since the origin is a non-degenerated singularity. Therefore close to the origin $\left(y_{1}, \ldots, y_{m-1}, y_{m}\right)$, where $y_{m}=x_{m}\left|f\left(y_{1}, \ldots, y_{m-1}, x_{m}\right)\right|^{1 / 2}$, is a system of coordinates as required.

Proposition 7.2. For every $r>0$ there exists a Morse function $\tau: \mathbb{R}^{m} \rightarrow \mathbb{R}$ such that:

(a) $\tau$ is $\Gamma$-invariant. 
(b) If $p$ is a minimum of $\tau$ then $\Gamma(p) \neq p$, that is to say $p$ does not belong to the hyperplane $x_{m}=0$.

(c) $|\tau(x)| \leq\|x\|^{2}$ on $\mathbb{R}^{m}$ and $\tau(x)=\|x\|^{2}$ on $\mathbb{R}^{m}-B(r)$.

For proving the foregoing proposition we need:

Lemma 7.3. There exists a smooth function $\rho: \mathbb{R} \rightarrow \mathbb{R}$ such that

(a) $\rho(t)=1$ if $t \geq 1, \rho(t)=-1$ if $t \leq 0, \rho(1 / 2)=0,0<\rho<1$ on $(1 / 2,1)$ and $-1<\rho<0$ on $(0,1 / 2)$. Moreover $\rho(1-t)=-\rho(t), t \in \mathbb{R}$, that $t$ is to say $\rho$ is anti-symmetrical with respect to $t=1 / 2$.

(b) $\rho^{\prime} \geq 0$ on $\mathbb{R}$ and $\rho^{\prime}>0$ on $(0,1)$. Moreover $\rho^{\prime}(1-t)=\rho^{\prime}(t), t \in \mathbb{R}$.

(c) $\rho^{\prime \prime}>0$ on $(0,1 / 2), \rho^{\prime \prime}<0$ on $(1 / 2,1)$ and $\rho^{\prime \prime}=0$ on $(\mathbb{R}-(0,1)) \cup\{1 / 2\}$. Moreover $\rho^{\prime \prime}(1-t)=-\rho^{\prime \prime}(t), t \in \mathbb{R}$.

Proof. Let $\varphi$ be a smooth function meeting the requirements of (c). Denote by $\varphi_{1}$ its primitive with initial condition $\varphi_{1}(0)=0$ and by $\varphi_{2}$ the primitive of $\varphi_{1}$ such that $\varphi_{2}(1 / 2)=0$.

Then $\varphi_{2}$ is constant and positive on $[1, \infty)$ while it is constant and negative on $(-\infty, 0]$. Moreover $\varphi_{2}(0)=-\varphi_{2}(1)$. The function $\rho=\left(\varphi_{2}(1)\right)^{-1} \varphi_{2}$ meets the requirements of the lemma (draw the graphics of $\varphi, \varphi_{1}$ and $\varphi_{2}$ ).

Corollary 7.4. Consider the function $\lambda$ defined by $\lambda(t)=t \rho(t)$. If $\lambda^{\prime}(c)=0$ then $c \in(0,1 / 2)$ and $\lambda^{\prime \prime}(c)>0$.

Proof of Proposition 7.2. First observe that if $\tau$ is like in Proposition 7.2 for $r=1$, then for any other $r>0$ it suffices to take $\tau_{r}(x)=r^{2} \tau\left(r^{-1} x\right)$.

Consider a function $\rho$ as in Lemma 7.3 and set $\tau(x)=x_{1}^{2}+\cdots+x_{m-1}^{2}+x_{m}^{2} \rho\left(\|x\|^{2}\right)$. Then $|\tau(x)| \leq\|x\|^{2}$ everywhere and $\tau(x)=\|x\|^{2}$ if $\|x\| \geq 1$. On the other hand an elementary computation making use of Corollary 7.4 shows that the singularities of $\tau$ are always nondegenerate and belong to the last axis, while the origin is a saddle.

Remark 7.5. As in one variable between two consecutive minima there always exists a maximum, the function $\lambda$ of Corollary 7.4 has a single singularity, which is a minimum. A more careful computation shows that function $\tau$ of the proof of Proposition 7.2 has just three singular points: a saddle and two minima. 


\section{REFERENCES}

[1] Bredon, G.E., "Introduction to compact transformation groups", Academic Press, Pure and Applied Mathematics, Vol. 46, 1972.

[2] Bing, R., A homeomorphism between the 3-sphere and sum of the solid horned spheres, Ann. of Math. (2). 56 (1952), 354-362.

[3] Hirsch, M.W., "Differential topology", Graduate Texts in Mathematics, No. 33. Springer-Verlag, New York-Heidelberg, 1976.

[4] Hirzebruch, F., Singularities and exotic spheres, Séminaire Bourbaki 314 (1966/67), 13-32.

[5] Kobayashi, S. and Nomizu, K., "Foundations of differential geometry Vol. I", Publishers, a division of John Wiley \& Sons, New York-London 1963.

[6] Kwasik, S. and Lee, K.B., Locally linear actions on 3-manifolds, Math. Proc. Cambridge Phil. Soc. 104 (1998) 253-260.

[7] Quinn, F., Ends of maps. III: dimension 4 and 5, J. Differential Geometry 17 (1982) 503-521.

[8] Turiel, F.-J. and Viruel, A., Finite $C^{\infty}$-actions are described by a single vector field, Rev. Mat. Iberoam. 30 (2014), 317-330.

[9] Wasserman, A., Equivariant differential topology, Topology 8 (1969), 127-150.

(F.J. Turiel) Departamento de Álgebra, Geometría y Topología, Facultad de Ciencias, CamPus de Teatinos, s/n, 29071-Málaga, Spain

E-mail address, F.J. Turiel: turiel@uma.es

(A. Viruel) Departamento de Álgebra, Geometría y Topología, Facultad de Ciencias, Campus de Teatinos, s/n, 29071-Málaga, Spain

E-mail address, A. Viruel: viruel@uma.es 